\title{
Heterotopic pregnancy in a spontaneous cycle
}

Published online: 26 October 2004

(C) Springer-Verlag Berlin / Heidelberg 2004

\begin{abstract}
Heterotopic pregnancy, defined as the simultaneous occurrence of intrauterine and extrauterine pregnancies, is rare in a natural cycle. Assisted reproductive techniques have resulted in an increased incidence. Identification of an intrauterine pregnancy can divert attention from the possibility of a concurrent ectopic pregnancy. We describe such a case with the educational features.
\end{abstract}

Keywords Heterotopic pregnancy - Ectopic pregnancy · Early pregnancy bleeding

\section{Introduction}

Heterotopic pregnancy, defined as the simultaneous occurrence of intrauterine and extrauterine pregnancies, is rare in a natural cycle. Assisted reproductive techniques have resulted in an increased incidence. Identification of an intrauterine pregnancy can divert attention from the possibility of a concurrent ectopic pregnancy. We describe such a case with the educational features.

\section{Case report}

A 41-year-old woman (gravida 4, para 0) with a history of four first trimester miscarriages presented at 5 -week gestation complaining of left-sided lower abdominal pain and vaginal bleeding. Physical examination revealed a stable patient with mild lower abdominal tenderness. Vaginal examination revealed mild cervical excitation and a uterine size compatible with 5-week gestation. Her haemoglobin was $13.4 \mathrm{gm} / \mathrm{dl}$ and serum hCG $6,653 \mathrm{U} / \mathrm{ml}$.

N. Mishra · A. Burnham

Department of Obstetrics and Gynaecology,

Frimley Park Hospital,

Frimley, Camberley, Surrey, UK

N. Mishra (

5 Lincolnshire Gardens, Warfield, Bracknell, RG42 3XB, UK

e-mail: nutanmishra@hotmail.com
A transvaginal ultrasound scan (TVS) showed a 10-mm intrauterine gestation sac with a yolk sac, but no fetal pole. There was free fluid in the pouch of Douglas (PoD).

Her serum hCG $48 \mathrm{~h}$ later was 253,942 U/ml. She continued to have vaginal bleeding and a repeat TVS showed an irregular 15-mm gestation sac with no yolk sac or fetal pole and a hypoechoic area of bleeding around the sac. A provisional diagnosis of a missed miscarriage was made with the possibility of molar proliferation in view of the high serum hCG.

The patient underwent an evacuation of retained products of conception (ERPC) with the histology subsequently confirming products of conception.

Serial serum hCG measurements post-ERPC showed an initial fall to $3,607 \mathrm{U} / \mathrm{ml}$ at $48 \mathrm{~h}$ and a subsequent rise to $6,273 \mathrm{U} / \mathrm{ml}$ at 1 week. She also had mild intermittent lower abdominal pain. A TVS showed an empty uterus and normal ovaries. There was a $3.5 \times 3.4-\mathrm{cm}$ complex mass adjacent to the left ovary with a small amount of fluid in the PoD. A 3-cm unruptured left ectopic pregnancy was confirmed at laparoscopy and a partial left salpingectomy performed. Histology confirmed an ectopic pregnancy.

\section{Discussion}

The histology provided confirmation of the simultaneous occurrence of intrauterine and extrauterine pregnanciesa heterotopic pregnancy in this patient. This condition is very rare in a natural cycle, with an estimated occurrence of one per 30,000 spontaneous pregnancies. Assisted reproductive technologies have caused an increase in the incidence.

Lack of early symptoms causes diagnostic confusion. Abdominal pain, adnexal mass, peritonism and an enlarged uterus are common features [1]. TVS is the most useful diagnostic tool [2]. Our patient was initially considered to have had a missed miscarriage. However, the serum hCG level of $253,942 \mathrm{U} / \mathrm{ml}$ raised a suspicion of trophoblastic proliferation. The easy detection of an intrauterine sac on TVS diverted our attention from a possible ectopic pregnancy. However, the persistently elevated serum hCG post-ERPC and her intermittent mild abdominal pain prompted a repeat TVS, which detected the ectopic mass. In retrospect, the first TVS, without showing the ectopic mass, had shown free fluid in the 
pouch of Douglas, a finding deemed indicative of the possibility of an ectopic pregnancy [3].

The surgical management in this patient was a laparoscopic salpingectomy. Most cases of heterotopic pregnancy are managed in this way. However, nonsurgical management by injection of potassium chloride in the gestation sac is described [4].

Heterotopic pregnancy, although rare, should be considered in patients with a persistently elevated hCG and abdominal pain post-ERPC. It is important to remember that the detection of an intrauterine pregnancy does not exclude the existence of an accompanying ectopic pregnancy.

\section{References}

1. Reece EA, Petrie RH, Sirmans MF, Finster M, Todd WD (1983) Combined intrauterine and extrauterine gestations: a review. Am J Obstet Gynecol 146:323-330

2. Louis-Sylvestre C, Morice P, Chapron C, Dubuisson JB (1997) The role of laparoscopy in the diagnosis and management of heterotopic pregnancies. Hum Reprod 12:1098-1099

3. Romero R, Kadar N, Castro D (1998) The value of adnexal sonographic findings in the diagnosis of ectopic pregnancy. Am J Obstet Gynecol 158:52-57

4. Fernandez H, Helaidier C, Doumere S, Fournet P, Olivenes F, Frydman R (1993) Nonsurgical treatment of heterotopic pregnancy: a report of six cases. Fertil Steril 60:428-432 